\title{
CP Violation and B mixing in CDF-II
}

\author{
A. Ruiz (representing CDF-II Collaboration) ${ }^{a}$ \\ ${ }^{a}$ Instituto de Física de Cantabria, \\ C.S.I.C.- Universidad de Cantabria \\ Santander, Spain
}

In March 2001, the Tevatron at Fermilab will start a new run at $\sqrt{s}=2.0 \mathrm{TeV}$, delivering an integrated luminosity of $2 \mathrm{fb}^{-1}$ in the first two years and more than $15 \mathrm{fb}^{-1}$ in the following years, before the start of LHC at CERN.

CDF has been upgraded to cope with the new physics program. We present here the prospects for measuring $\mathrm{B}$ mixing and $\mathrm{CP}$ violation during the upcoming Run.

\section{Introduction}

In the following years the Tevatron at Fermilab will be the sole hadronic machine able to produce $b$ mesons and baryons competitively with the $b$ factories Moreover, it will be the unique laboratory to explore $B_{s}^{0}$ mesons and $\Lambda_{b}$ baryons which will not be produced at the $b$ factories operating at the upsilon $\Upsilon(4 S)$ resonance.

The currently named Run II will provide 20 times greater luminosity than Run I, at a center of mass energy of $2 \mathrm{TeV}$. It is expected to reach an integral luminosity of $2 \mathrm{fb}^{-1}$ in the first two years in each of the two experiments ( CDF and D0), starting March 2001. A second step will provide a total luminosity higher than $15 \mathrm{fb}^{-1}$ before the turn-on of the LHC.

The amount of $b \bar{b}$ pairs per year in the first step of Run II will be about $10^{11}$ with a big background ( about three orders of magnitude higher) of events. A complex and very efficient trigger system has been designed to cope with this scene.

The experiment CDF has been upgraded[1] in order to maintain the occupancies of the Run $I$ and to improve its performance, providing a new tracking system and rising the acceptance of the global detector. Also, as a beyond baseline project[2] the installation of a Time of Flight detector system was approved which considerably improves the identification capabilities and is significantly important in the b physics program.

From 1991-1995 CDF has collected $110 p b^{-1}$ at $\sqrt{s}=1.8 \mathrm{TeV}$. Several studies were made of $B^{0} B^{0}$ oscillations using $B_{d}$ and $B_{s}$ mesons [3]. The measure of mixing asymmetries using several tagging methods produced an average result of $\Delta\left(m_{d}\right)=0.425 \pm 0.026 \pm 0.025 p^{-1}$ and a limit of $\Delta\left(m_{s}\right)>5.8 \mathrm{ps}^{-1}$ at $95 \%$ C.L. The B flavor tagging at the production time was based on lepton tag or jet charge tag on the side of the opposite $\mathrm{B}$, or charge correlation on the same side.

Applying the three methods of flavor tagging, CDF produced the best direct measurement of CP violation in the $B$ system, with a result of the CKM parameter $\sin (2 \beta)=0.79 \pm 0.39 \pm 0.16[4]$, which translates to be positive at $93 \%$ C.L.

The introduction of the new Time of Flight system will increase the flavor tagging effectiveness of CDF by applying new methods based on Kaon tagging. Moreover, a new silicon layer close to the interaction point will improve the time resolution of the $b$ hadron decay. A new trigger based on the displacement of the interaction point of the decay products of $b$ hadrons will allow the efficiency and signal/background of its hadronic decay to improve considerably.

In the following sections we will report on the main upgrades of the detector and trigger system, as well as the Havor tagging methods. In the last sections we will give an overview of the studies which have been made to forecast the future possibilities of $B^{0}$ oscillations and $\mathrm{CP}$ violation measurements in the Run II of CDF. 


\section{The CDF upgrade}

\subsection{The Tevatron upgrade}

The accelerator complex of Fermilab has been upgraded, mainly with the inclusion of a new "Main Injector" which will increase in a factor three over the RUN I the production rate of antiprotons. Moreover the number of proton bunches will increase to 36 in a first stage and 108 in a second stage. The beams will cross every 396 nanoseconds in the first stage and every 132 nanoseconds in the second one. The number of overlapping interactions expected by crossing goes from 2 to 6 on average.

\subsection{Detector upgrade}

The CDF tracking system has been completely changed and includes[1], [2]:

- A new 5 silicon layer system (SVX-II) extending from a radius of $2.4 \mathrm{~cm}$. to $10.7 \mathrm{~cm}$. The layers will have double side readout allowing 3-dimensional reconstruction of the tracks The pitch will be 60 microns and the resolution by point 12 microns. The acceptance in rapidity will be $|\eta|<2$

- A new 2 silicon layer system (ISL) with the same acceptance as the SVX-II system, with a radius of $20 \mathrm{~cm}$. to $28 \mathrm{~cm}$., with double side readout, 110-146 microns pitch and 16 microns resolution per point.

- a new open cell drift chamber (COT) with a radius of $44 \mathrm{~cm}$. to $132 \mathrm{~cm}$. and acceptance of $|\eta|<1$. The cell drift times will be less than 132 nsec. The COT will consist in 96 layers grouped in 8 superlayers, 4 of them with $r-\phi$ readout and the other 4 with $\mathrm{z}$ readout. The resolution per point will be 180 microns and will provide $\mathrm{dE} / \mathrm{dx}$ measurement.

- "beyond the baseline", a new silicon layer (L00) at a radius of $1.6 \mathrm{~cm}$. with singleside readout and 50 microns pitch, with a very hard radiation multi guard ring design

The impact parameter resolution expected is

$\sigma(d)=5.5+17 \mu \mathrm{m} / p_{T}\left(p_{T}\right.$ in $\left.\mathrm{GeV}\right)$
The time resolution for $\mathrm{B}$ decays is $60 \mathrm{fsec}$ without the L00 and 45 fsec. with L00.

The momentum resolution expected is

$\sigma^{2}\left(p_{T}\right)=\left(0.0009 p_{T}\right)^{2}+0.0066^{2}$

The muon system will be doubled in coverage up to $|\eta|<2$ and the drift chambers will operate in proportional mode to cope with the high rates of Run II.

A new scintillating tile plug calorimeter will be included in the range $1<|\eta|<2$. The expected energy resolution is:

$\sigma\left(E_{T}\right)=\left(10 \% E_{T}\right)+1.0 \mathrm{GeV}$

A new Time of Flight system (TOF) has been installed between the COT and the superconducting solenoid. The readout will be made by 19 stage fine-mesh Hamamatsu PMT's operating in a strong magnetic field of 1.4 Tesla. The system has been designed to give a timing resolution of 100 picoseconds, which will allow a $2 \sigma$ separation of Kaons and pions for $p<1.6 \mathrm{GeV} / \mathrm{c}$ being complementary to the identification based on $\mathrm{dE} / \mathrm{dx}$ from the COT.

\subsection{The new trigger system}

A new pipelined trigger system with a 3-level architecture will be installed. The Level-1 system will work at $50 \mathrm{KHz}$ and, besides the usual lepton trigger, will include an extremely fast track trigger (XFT) for tracks with $\left|P_{T}\right|>1.5 \mathrm{GeV}$, with a performance:

$\sigma\left(p_{T}\right) / p_{T}^{2}=0.012(\mathrm{GeV} / \mathrm{c})^{-1}$

$\Delta(\phi)<0.0015 \mathrm{rad} .($ at $\mathrm{R}=106 \mathrm{~cm}$.)

The track information from XFT will be distributed (XTRP) to the Level-1 muon, calorimeters and track to conform the Global Level 1 trigger.

The Level-2 will work at a rate of $300 \mathrm{~Hz}$. It will include a displaced track trigger (SVT) which uses the information of XTRP and the SVX. Its performance will be:

$\sigma\left(p_{T}\right) / p_{T}^{2}=0.003(G e V / c)^{-1}$

$\Delta(\phi)=0.001 \mathrm{rad} .($ at $\mathrm{R}=106 \mathrm{~cm}$. 
$\Delta(d)=35 \mu \mathrm{m}$ (impact parameter)

Many asynchronous subsystems will work, including the most interesting ones for B physics as two-tracks (e.g. $B \rightarrow \pi \pi, K \pi, K K$ ), all hadronic $\mathrm{B}$ decays and soft lepton plus XFT tracks.

The Level-3, with a rate of $30 \mathrm{~Hz}$. will be made by software. The limit dead time will be less than $10 \%$ at full luminosity.

\section{Flavor tagging}

The tag of the $\mathrm{B}$ flavor at production and decay time is essential to analyze $\mathrm{B}$ mixing and CPviolation. CDF will use several methods extending the old ones used in Run I, indicated in the Introduction. The "figure of merit" of the tagging methods is the factor $\epsilon D^{2}$, where $\epsilon$ is the real tagging efficiency and $\mathrm{D}$ is the dilution factor defined as $2 \mathrm{P}-1$, where $\mathrm{P}$ is the probability of a correct flavor tag. The statistical error on the determination of a CP asymmetry is inversely proportional to the square root of the effective tag $\epsilon D^{2}$.

The inclusion of the TOF enhance considerably the effective tag both in the opposite-side and the same-side, by using the Kaon identification tag (which is possible in about the $57 \%$ of the cases for the opposite side)[2]. As it is shown in Table 1, the inclusion of the TOF will be crucial for some channels. Also the expected reconstructed signal is shown, calculated from data of Run I scaled to the new trigger efficiency and luminosity

\section{Prospective for B mixing in Run II}

In addition to improving the measurement of $\Delta\left(m_{d}\right)$, CDF-II will be able to measure $B_{s}^{0} \overline{B_{s}^{0}}$ mixing by using the hadronic decay channels: $B_{s} \rightarrow D_{s}^{+} \pi^{-}$and $B_{s} \rightarrow D_{s}^{+} \pi^{-} \pi^{-} \pi^{+}$.

With an expected reconstructed signal $(\mathrm{N})$ before flavor tagging of more than 23000 events and an effective tagging $\epsilon D^{2}$ of $11.3 \%$ the sensitivity to $x_{s}=\Delta\left(m_{s}\right) \tau\left(B_{s}^{0}\right)$ goes from 42 to 63 depending on the signal/background relation, which is expected to be between $1 / 2$ and 2[2], [5].

Figure 1 shows the significance calculated from

$\operatorname{Sig}\left(x_{s}\right)=\sqrt{\frac{N \epsilon D^{2}}{2}} e^{-\left(x_{s} \sigma_{t} / \tau_{s}\right)^{2} / 2} \sqrt{\frac{S}{S+B}}$

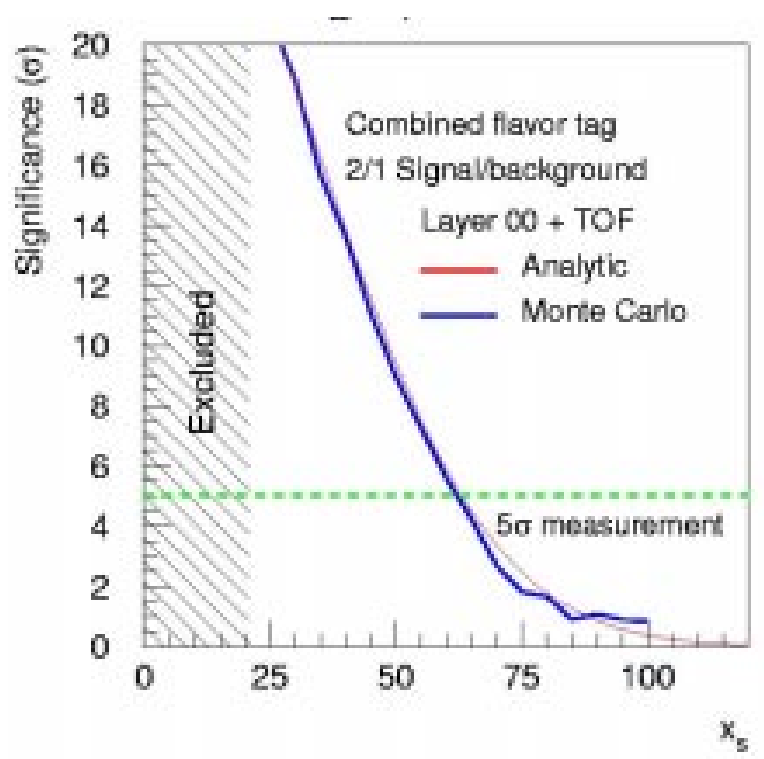

Figure 1. Significance distribution for $x_{s}$

in the case of a rate Signal(S) over Background(B) equal to 2. The timing resolution $\sigma_{t}$ will be 60 fsec. without the L00 and 45 fsec with L00.

Another possibility will be to continue study oscillations in the semileptonic decay channels $B_{s} \rightarrow D_{s}\left(D_{s}^{*}\right) l \nu$, with $D_{s} \rightarrow \Phi \pi, K^{*} K$, using the lepton + SVT track trigger. In fact the sensitivity is much lower than in the case of hadronic decays. However, the big signal will be very useful for other studies as the measurement of $\Delta(\Gamma)[6]$.

\section{Prospective for CP violation in Run II}

\subsection{Measurement of $\sin (2 \beta)$}

The golden channel to measure the beta angle of the unitarity triangle will continue to be $B_{d}^{0}, \overline{B_{d}^{0}} \rightarrow J / \Psi K_{s}$, where the CP asymmetry is given by:

$A_{C P}(t)=\frac{N\left(B_{d}^{0}\right)-N\left(\overline{B_{d}^{0}}\right)}{N\left(B_{d}^{0}\right)+N\left(\overline{B_{d}^{0}}\right)}=-\sin (2 \beta) \sin \left(\Delta\left(m_{d}\right) t\right)$ 
Table 1

Effective tag $\epsilon D^{2}(\%)$

\begin{tabular}{llll}
\hline tag & $B^{0} \rightarrow J / \psi K_{S}^{0}$ & $B^{0} \rightarrow J / \psi \Phi$ & $B^{0} \rightarrow D_{s}^{-} \pi^{+}$ \\
\hline Soft Lepton Tagging( $\mu$ ) & 1.0 & 1.0 & 1.0 \\
Soft Lepton Tagging(e) & 0.7 & 0.7 & 0.7 \\
Jet Charge Tagging & 3.0 & 3.0 & 3.0 \\
Opposite Side Tagging (Kaon) & 2.4 & 2.4 & 2.4 \\
Same Side Tagging(pion) & 1.9 & & \\
Same Side Tagging(kaon) & & 2.6 & 4.2 \\
\hline Total & $9.0 \%$ & $9.7 \%$ & $11.3 \%$ \\
Total(without TOF) & $6.1 \%$ & $4.9 \%$ & $5.7 \%$ \\
Signal size expected before tagging in $2 \mathrm{fb}^{-1}$ & 30000 & $>4000$ & 10600 \\
\hline
\end{tabular}

From it the resolution on the $\sin (2 \beta)$ measurement will be:

$\sigma(\operatorname{sen}(2 \beta)) \simeq e^{-\left(x_{d}^{2} \sigma_{t}^{2} / \tau_{d}^{2}\right)} \sqrt{\frac{1+4 x_{d}^{2}}{2 x_{d}}} \frac{1}{\sqrt{\epsilon D^{2} N}} \sqrt{\frac{S+B}{B}}$

With a signal of 30000 reconstructed events, the expected value will be $\sigma(\sin (2 \beta))=0.043[7]$

\subsection{Measure of $\gamma$}

There exist at least three alternative methods of measuring the unitarity angle $\gamma$.

- The first one[8] uses the decay $B_{s}^{0}, \overline{B_{s}^{0}} \rightarrow$ $D_{s}^{ \pm} K^{\mp}$ using the fact that the weak amplitude of mixing is almost real, as it is the decay amplitude of $B_{s}^{0} \rightarrow D_{s}^{-} K^{+}$, while the decay $\overline{B_{s}^{0}} \rightarrow D_{s}^{-} K^{+}$has a phase which is approximately $\gamma$..

In fact using the total number of events decaying to the two final states, the CP asymmetry is:

$A_{C P}(t)=\frac{N\left(B_{s}^{0}\right)-N\left(\overline{B_{s}^{0}}\right)}{N\left(B_{s}^{0}\right)+N\left(\overline{B_{s}^{0}}\right)}$

$=-\sin (\gamma) \sin \left(x_{s} t\right) \cos (\delta) \sqrt{1-R^{2}}$

where $\delta$ is a strong phase difference between the two decay and $\mathrm{R}=\frac{\rho^{2}-1}{\rho^{2}+1}, \rho$ being the ratio of the decay amplitudes of $B_{s}^{0}$ and $\overline{B_{s}^{0}}$

In Figure 2 is shown the expected error of $\sin (\gamma+\delta)$ as a function of the reconstructed number of events for $\mathrm{S} / \mathrm{B}=1 / 6$ ( upper part) and $\mathrm{S} / \mathrm{B}=1$ ( lower one). The points correspond to $2,5,10$ and $20 \mathrm{fb}^{-1}$ of integrated luminosity

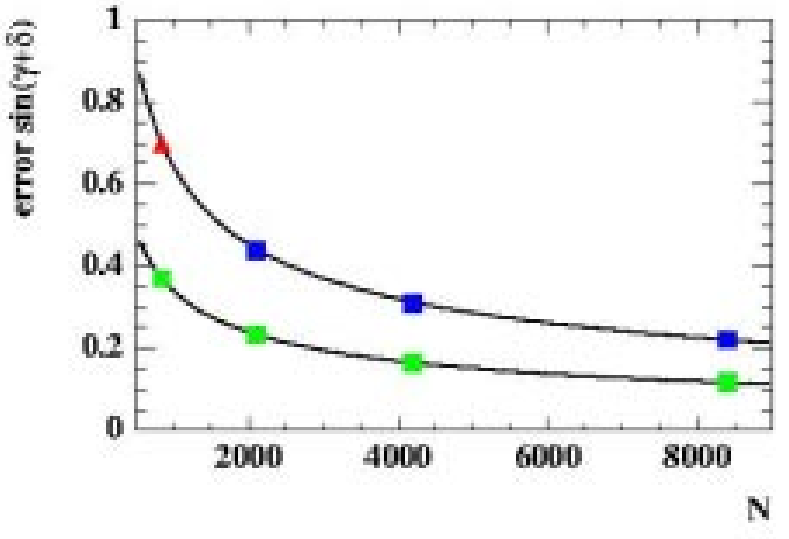

Figure 2. $\sin \gamma$ error ( see text)

- Other method will use a fit on the CP violating observables $B_{d}^{0}, \overline{B_{d}^{0}} \rightarrow \pi^{+} \pi^{-}$, $B_{s}^{0}, \overline{B_{s}^{0}} \rightarrow K^{+} K^{-}$and $B_{d}^{0}, \overline{B_{d}^{0}} \rightarrow J / \psi K_{s}^{0}$.

Due to penguin pollution effects, the foreseen channel to measure the $\alpha$ angle of the unitarity triangle, $B_{d}^{0}, \overline{B_{d}^{0}} \rightarrow \pi^{+} \pi^{-}$, is no 
longer considered as the best one, but, assuming U spin symmetry, it is possible to relate the two channels $B_{d}^{0}, \overline{B_{d}^{0}} \rightarrow \pi^{+} \pi^{-}$and $B_{s}^{0}, \overline{B_{s}^{0}} \rightarrow K^{+} K^{-}$. The Penguin vs Tree amplitudes differences between those two channels are entirely dominated by CKM matrix elements.

Defining the amplitudes of decay to a final state f:

$A_{f}=<f|T| B^{0}>, \overline{A_{f}}=<f|T| \overline{B^{0}}>$

and the ratio $\lambda_{f}=\frac{\overline{A_{f}}}{A_{f}}$, the $\mathrm{CP}$ asymmetry is given, in the case of $\Delta(\Gamma) \simeq 0$ and no $\mathrm{CP}$ violation in the mixing, by:

$A_{C P}(t)=\frac{1-\left|\lambda_{f}\right|^{2}}{1+\left|\lambda_{f}\right|^{2}} \cos \left(\Delta_{m} t\right)$

$+\frac{2 \operatorname{Im} \lambda_{f}}{1+\left|\lambda_{f}\right|^{2}} \sin \left(\Delta_{m} t\right)$

which is written as $A_{\operatorname{dir}} \cos \left(\Delta_{m} t\right)+$ $A_{m i x} \sin \left(\delta_{m} t\right)$

R. Fleischer[9], using U spin symmetry, has recently written a system of four equations of $A_{m i x}\left(K^{+} K^{-}\right), A_{m i x}\left(\pi^{+} \pi^{-}\right)$, $A_{\text {dir }}\left(K^{+} K^{-}\right), A_{\text {dir }}\left(\pi^{+} \pi^{-}\right)$, depending on the ratio of Penguin-tree matrix elements, the phase of the ratio of hadronic matrix elements and the weak phases of the unitarity triangle $\gamma$ and $\beta$.

CDF will collect more than 5000 reconstructed events on the channel $B_{d}^{0}, \overline{B_{d}^{0}} \rightarrow$ $\pi^{+} \pi^{-}$and about 10000 events on the channel $B_{s}^{0}, \overline{B_{s}^{0}} \rightarrow K^{+} K^{-}$.

Using the value of $\beta$ measured as a constraint, some analyses[10] have shown that it is possible to fit the above equations to measure $\gamma$ better than $10^{0}$ with $2 \mathrm{fb}^{-1}$

- The third method to measure $\gamma$ is based on measuring the decay rates $B^{ \pm} \rightarrow$ $D^{0}\left(\overline{D^{0}}\right) K^{ \pm}$using final states common to $D^{0}, \overline{D^{0}}$ which are not $\mathrm{CP}$ eigenstates[12].
These measurements don't present problems related to penguin decays, time dependence nor flavor tagging.

The method[11] consists in measuring the branching ratios:

$d\left(f, f^{\prime}\right)=B R\left(B^{+} \rightarrow K^{+}\left(f, f^{\prime}\right)\right)$ and $\bar{d}\left(f, f^{\prime}\right)=B R\left(B^{-} \rightarrow K^{-}\left(\bar{f}, \overline{f^{\prime}}\right)\right)$

using as final states $f=K^{-} \pi^{+}, f^{\prime}=$ $K^{-} \pi^{+} \pi^{+} \pi^{-}$

The expected number of reconstructed events for each channel is about 100 for an integrated luminosity of $2 \mathrm{fb}^{-1}$. Some preliminary studies indicate a resolution on $\gamma$ of about $20^{\circ}[12]$

\subsection{Other studies}

The channel $B_{s}^{0} \rightarrow J / \Psi \Phi \rightarrow \mu^{+} \mu^{-} K^{+} K^{-}$can be used to measure the $(\Delta(\Gamma) / \Gamma))_{B_{s}^{0}}$, where $\Delta(\Gamma)$ is the difference between the heavy and light weak eigenstates, which are, in a close approximation, the CP even and odd states.

The angular distribution of the decay products allows for a separation of the CP eigenstates. In fact the $\mathrm{CP}$ even state angular distribution goes as $3 / 8\left(1+\cos ^{2} \theta_{T}\right)$, while the $\mathrm{CP}$-odd goes as $3 / 4\left(\sin ^{2} \theta_{T}\right)$, where $\theta_{T}$ is the transversity angle [13].

The number of reconstructed events in $2 \mathrm{fb}^{-1}$ is expected to be more than 4000[14]. A preliminary analysis[15] has shown that it is possible to measure $(\Delta(\Gamma) / \Gamma))_{B_{s}^{0}}$ with a precision of 0.051 ( assuming, from current estimations, a value of $(\Delta(\Gamma) / \Gamma))_{B_{s}^{0}}=0.15$ and a fraction of CP-even state of 0.771 ( as was fitted in Run I) )

\section{Conclusions}

CDF has a rich program to exploit the new data of Run II at Fermilab. Among the B physics goals are: the measurements of $B_{d}^{0}$ and $B_{s}^{0}$ oscillations, with a sensitivity up to $x_{s}=63$, and the measurements of the $\alpha, \beta$ and $\gamma$ angles of the unitarity matrix. The expected resolution on $\sin (2 \beta)$ can reach the value of 0.043 , and the $\gamma$ angle can be measured up to $10^{\circ}$, with an integrated luminosity of $2 \mathrm{fb}^{-1}$. The upgraded CDF detector will establish a competitive program with the B facto- 
ries, with the advantage of being able to analyse, at the same time, the $B_{d}^{0}$ and $B_{s}^{0}$ physics.

\section{Acknowledgements}

I want to thank the B group of the CDF, in particular the chairmen M.Paulini and B.Wicklund for their support in the preparation of this talk.

I also want to thank the organizers of the Conference for their hospitality and good work.

This work was supported by the CICYT (Comisión Interministerial de Ciencia y Tecnología, Spain), under the project AEN99-0950

\section{REFERENCES}

1. The CDFII Collab., The CDFII Detector Technical Design Report, Fermilab Pub 96/390-E

2. The CDFII Coll., Proposal P-909 (1998) and Update (1999), postscripts files on Web pages:

www.cdf.fnal.gov/upgrades/btb.prpoposal.ps, www.cdf.fnal.gov/upgrades/btb_update_jan99.ps, M.Jones, J.Kroll, C.Chen,

CDF Internal note (1999)

3. F.Abe et al, Phys. Rev. D60, 112004 (1999),

Phys. Rev. D60, 072003 (1999),

Phys. Rev. D60, 051101 (1999),

Phys. Rev. Lett 82, 3576 (1999),

Phys. Rev. D59,032001 (1999),

Phys. Rev.Lett.80,2057 (1998)

4. T. Affolder et al.,

Phys. Rev. D61,072005 (2000)

5. M.Jones,

Contribution to the "Workshop on B Physics at Tevatron. RunII and beyond", Fermilab (2000)

6. M.Tanaka,

Contribution to the "Workshop on B Physics at Tevatron. RunII and beyond", Fermilab (2000)

7. B.Wicklund,

Contribution to the "Workshop on B Physics at Tevatron. RunII and beyond", Fermilab (2000)

8. S.Bailey and P.Maksimovic,
Contribution to the "Workshop on B Physics at Tevatron. RunII and beyond", Fermilab (2000)

9. R. Fleischer,

Phys. Lett. B459, 306 (1999)

10. F.Wurthwein, Contribution to the "Workshop on B Physics at Tevatron. RunII and beyond", Fermilab (2000), C. Blocker and F. Wurthwein, Contribution to the "Workshop on B Physics at Tevatron. RunII and beyond", Fermilab (2000)

11. D.Atwood, I.Dunietz and A. Soni, Phys. Rev. Lett. 78 (1997), 3257

12. A. Cerri, G.Punzi and G.Signorelli, CDF Internal note (2000)

13. I.Dunietz et. al., Phys. Lett. B369 (1996)

14. W.Wester, CDF Internal note (2000)

15. F.Azfar, L.Lyons, M.Martin, C.Paus and J.Tseng, Contribution to the "Workshop on B Physics at Tevatron. RunII and beyond", Fermilab (2000) 